\title{
Thermally activated escape rate for the Brownian motion of a fixed axis rotator in a double well potential for all values of the dissipation
}

\author{
William T. Coffey \\ Department of Electronic and Electrical Engineering, Trinity College, Dublin 2, Ireland \\ Yuri P. Kalmykov ${ }^{\text {a) }}$ \\ Groupe de Physique Moléculaire, MEPS, Université de Perpignan, 52, Avenue Paul Alduy, 66860 Perpignan \\ Cedex, France \\ Sergey V. Titov \\ Institute of Radio Engineering and Electronics of the Russian Academy of Sciences, Vvedenskii Square 1, \\ Fryazino, Moscow Region, 141190, Russian Federation
}

(Received 2 December 2003; accepted 19 February 2004)

\begin{abstract}
The extension of the Kramers theory of the escape rate of a Brownian particle from a potential well to the entire range of damping proposed by Mel'nikov and Meshkov [J. Chem, Phys. 85, 1018 (1986)] is applied to the rotational Brownian motion of fixed axis rotators in a double well cosine potential. The procedure yields an expression for the Kramers escape rate valid for all values of the dissipation including the very low damping (VLD), very high damping (VHD), and crossover regimes. This equation provides a good asymptotic estimate of the correlation time $\tau_{\|}$of the longitudinal dipole moment correlation function calculated by solving the underlying Langevin equation using the matrix-continued fraction method. Moreover, for low barriers, where the Mel'nikov and Meshkov approach is not applicable, analytic equations for $\tau_{\|}$in the VLD and VHD limits are derived and a simple extrapolating equation that is valid for all values of the damping is proposed. (C) 2004 American Institute of Physics. [DOI: 10.1063/1.1703525]
\end{abstract}

\section{INTRODUCTION}

The Brownian motion in a periodic potential is of interest in the solution of a variety of physical problems involving a relaxation process. Among the most prominent of these are dielectric relaxation of and the dynamic Kerr effect of nematic liquid crystals, magnetic relaxation of single domain ferromagnetic particles, dynamic response of Josephson tunneling junctions, transport phenomena in semiconductors, etc. ${ }^{1-3}$

One of the most important characteristics associated with the Brownian motion in a potential well is the greatest relaxation time or the time required to escape the well. The greatest relaxation time is essentially the inverse of the smallest nonvanishing eigenvalue $\lambda_{1}$ of the characteristic equation or secular determinant of the relevant dynamical system. Moreover, if the overbarrier mode characterized by $\lambda_{1}$ dominates the relaxation process as is always so for symmetric potential wells, the escape time $\left(\lambda_{1}^{-1}\right)$ will be closely approximated in the high barrier limit by the integral relaxation time. This time is the area under the relaxation function of the appropriate dynamic variable. ${ }^{2}$ In linear response, the integral relaxation time is identical to the correlation time of the corresponding autocorrelation function. However, for asymmetric potentials such as will arise from the imposition of a strong external field, it is not always possible to identify the integral relaxation time with the escape time. ${ }^{2}$ As far as the calculation of $\lambda_{1}$ is concerned, the secular equation may

${ }^{a}$ Corresponding author. be generated by averaging the appropriate Langevin equation over its realizations yielding the differential-recurrence equations governing the decay functions of the system (which is analogous to the use of matrix mechanics in quantum theory). Alternatively, one may expand the solution of the associated probability density diffusion equation (usually the specialized form of the Fokker-Planck equation known as the Klein-Kramers equation which applies to separable and additive Hamiltonians) in Fourier series in the position and velocity variables. ${ }^{1,2}$ In each of the two methods, the secular determinant results from truncation of the set of differentialrecurrence relations at a number large enough to achieve convergence of the resulting set of simultaneous ordinary differential equations. Alternatively, if the problem is represented in the frequency domain so that the more powerful continued fraction method may be used (which is very effective from a computational point of view), many convergents must be taken. ${ }^{1,4}$ Thus the smallest nonvanishing eigenvalue $\lambda_{1}$ is not in general available in closed form as it is always rendered as the smallest root of a high-order polynomial equation. Hence it is difficult to compare $\lambda_{1}$ so determined with experimental observations of the greatest relaxation time or the relaxation rate. Fortunately (noting that $\lambda_{1}$ for sufficiently high barriers has exponential dependence on the barrier height), a way of overcoming this difficulty is to utilize an ingenious method originally proposed by Kramers ${ }^{5}$ in connection with thermally activated escape of particles out of a potential well. His idea, motivated by the fluctuationdissipation theorem, ${ }^{2}$ is to calculate the prefactor in an Arrhenius like equation for the reaction rate $\Gamma$, viz., 


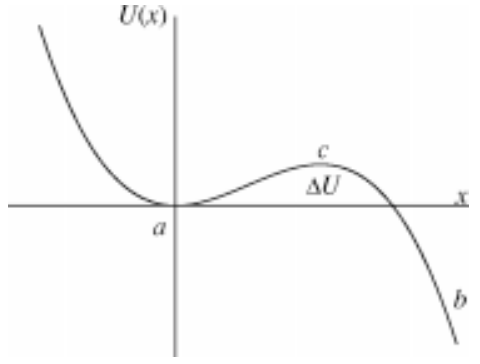

FIG. 1. Single well potential function.

$$
\Gamma=A \frac{\omega_{a}}{2 \pi} e^{-\beta \Delta U}
$$

as a function of the parameter $A$. That parameter represents the interchange of energy between the reacting particles and their surroundings or heat bath (for reviews of applications of Kramers' method, see Refs. 6 and 7).

Equation (1) for the Kramers escape rate $\Gamma$ (reaction velocity for chemical reactions) pertains to a model of a chemical reaction where rare members of an assembly of Brownian particles that are initially trapped in a potential well at $a$ (see Fig. 1) may subsequently under the influence of thermal agitation escape over a high $(\Delta U \gg k T)$ barrier at $c$ and descend to the bottom of a very deep potential well $b$ and so never return to $a$. Thus we model a chemical reaction (ignoring quantum effects) by introducing a reaction coordinate $x$ such that $x=a$ in species $a$ and $x=b$ in species $b$ (the product state). The reaction is modeled by thermally activated diffusion over the boundary $c$ (the transition state) between the two distinct states. The frequency $\omega_{a} / 2 \pi$ which is the frequency of oscillation of a particle in the potential well centred on $a$ is called the attempt frequency. The original Arrhenius equation which is obtained when $A=1$ [corresponding to transition state theory (TST)] assumes perpetual thermal equilibrium everywhere at temperature $T$. Thus no account is taken of nonequilibrium effects due to the leaking of particles over the potential barrier at $c$. In reality the Maxwell-Boltzmann distribution no longer holds in the vicinity of the transition state $c$ because the fluctuation dissipation theorem describing the coupling of the reacting particles to their surroundings or heat bath is violated by the Arrhenius equation.

Kramers ${ }^{5}$ overcame this difficulty by writing the diffusion equation (Klein-Kramers equation) in phase space describing the evolution of the phase-space distribution function underlying the Langevin equation for a Brownian particle. He then obtained asymptotic solutions (the Kramers escape rate) for the smallest nonvanishing eigenvalue $\lambda_{1}$ of the Klein-Kramers equation in the limits of very small and intermediate to high dissipative coupling to the bath. These solutions, which are valid for high barriers $(\Delta U \gg k T)$ so that the concept of an escape rate is valid, provide closed form expressions for the reaction rate and its inverse the greatest relaxation time $\tau \approx \lambda_{1}^{-1}$ which may be easily compared with experiment.

As far as the various dissipation regimes are concerned, the very low damping (VLD) regime is defined by Kramers as follows. First, he supposes that the energy trajectories of the Brownian particle in the well differ but little from those of the undamped periodic motion in the well. Thus the trajectories are closed except for a particular trajectory with energy corresponding to the barrier point energy associated with the transition state $c$. Particles on this particular trajectory known as the separatrix (between the bounded motion in the well and the motion outside) may either escape the well or else return to the depths of the well. The VLD regime is then defined by the condition that the energy loss per cycle $\Delta E$ of the almost periodic motion of a particle having the saddle-point energy is much less than the thermal energy $k T$. This condition, recognized on solution of the VLD problem, ${ }^{5,7}$ means that $A \ll 1$ in Eq. (1). Thus the escape rate vanishes in the absence of coupling to the heat bath so reconciling reaction rate theory with the fluctuation-dissipation theorem. The condition may also be written formally as

$$
\Delta E=\beta S\left(E_{c}\right) \simeq \beta \Delta U / \omega_{c} \ll k T .
$$

Here $S\left(E_{c}\right)$ is the action on the barrier point energy trajectory and $\beta$ is the dissipation constant. Moreover, the action of a harmonic oscillator of energy $\Delta U$ and angular frequency $\omega_{c}$ has been used to represent $S\left(E_{c}\right)$. Kramers obtained his solution for the VLD escape rate by first writing the KleinKramers equation in energy angle variables. He then eliminates the fast angle variable by averaging along the energy trajectories so obtaining a diffusion equation in the slowly varying energy variable. In his derivation, the coupling between the Liouville and diffusion terms in the KleinKramers equation is effectively ignored because the motion is supposed almost conservative.

The intermediate to high damping (IHD) regime occurs when the energy loss per cycle is sensibly greater than $k T$ :

$$
\Delta E \sim \beta \Delta U / \omega_{c}>k T .
$$

It is now impossible to ignore the coupling between the Liouville and dissipative terms. Here Kramers obtained asymptotic solutions for the escape rate by linearizing the Langevin equation about the point $c$. Such a procedure is legitimate because the region of departure from the Maxwell-Boltzmann distribution is now so limited in spatial extent because of the relatively high damping, that it lies well inside the region in which it is permissible to linearize the Langevin equation. In other words he represents the potential in the vicinity of $c$ by an inverted harmonic oscillator potential. He then solved the corresponding linearized (in the position variable) Klein-Kramers equation by supposing that the solution is quasistationary since escape over the barrier is an exponentially slow process obtaining

$$
A=\sqrt{1+\frac{\beta^{2}}{4 \omega_{c}^{2}}}-\frac{\beta}{2 \omega_{c}} .
$$

This equation contains the TST theory $(A=1)$ as a limiting case when

$$
\beta / \omega_{c} \ll 1
$$

provided the IHD condition [Eq. (3)] is also satisfied. Hence combining Eqs. (3) and (5) we expect the Arrhenius equation to approximately hold in the range of damping, 
$k T \omega_{c} / \Delta U<\beta \ll \omega_{c}$,

and not for zero damping as expected by naively taking the limit of Eq. (4). The IHD solution also contains as a limit the very high damping (VHD) solution, where $\Delta E \gg k T$. This solution amounting to $A=\omega_{c} / \beta$ was also obtained by Kramers by constructing the approximate Smoluchowski equation governing the evolution of the distribution function in configuration space only. That equation is valid if the effect of inertia of a particle on its nonequilibrium dynamics is ignored.

Kramers was, however, unable to find asymptotic solutions for the crossover regime, $\Delta E \sim k T$, where the coupling between the Liouville and dissipative terms in the KleinKramers equation enters so that one may no longer ignore the Liouville term as was done in the very low damping regime. This problem, named the Kramers turnover problem, was solved nearly 50 years later by Mel'nikov and Meshkov. ${ }^{8,9}$ They gave an integral formula bridging the VLD and TST solutions. Their solution consists of an integral which when multiplied by the TST result yields a formula which reduces to the VLD formula as $\beta \rightarrow 0$. Now the TST result is a particular case of the IHD formula Eq. (4) provided the condition embodied in Eq. (6) is satisfied. Thus they postulate from heuristic reasoning, essentially appealing to continuity that a formula valid for all values of the damping may be given by simply multiplying the general IHD result by their bridging integral. Mel'nikov ${ }^{8}$ has further extended the bridging integral method to take into account quantum effects in a semiclassical way. Moreover, Grabert ${ }^{10}$ and Pollak et al. ${ }^{11}$ later presented a complete solution of the Kramers turnover problem and have shown that the Mel'nikov and Meshkov universal formula can be obtained without ad hoc interpolation between the weak and strong damping regimes. In the semiclassical limit, the latter theory was extended to the quantum regime by Rips and Pollak. ${ }^{12}$

As far as the verification of the universal turnover formula of Mel'nikov and Meshkov is concerned, very few comparisons of that formula with exact calculations based on either the solutions of the Klein-Kramers equation or on numerical simulations of the Brownian dynamics have ever been given. Exceptions are the comparison of the universal turnover formulas with the numerical results for the escape out of a single well, which were given in Refs. 13 and 14 and the study of the one-dimensional translational Brownian motion in a periodic potential undertaken by Ferrando and co-workers. ${ }^{15,16}$ Another exception is the treatment of the same one-dimensional problem and its generalization to diffusion on a surface which was undertaken by Pollak and collaborators in Refs. 17-19. Examples of the exact treatment of rotational Brownian motion problems are even fewer. Pastor and $\mathrm{Szabo}^{20}$ tested the Mel'nikov-Meshkov formula in the context of a linear molecule in a uniaxial potential and Coffey and co-workers ${ }^{7,21}$ extended the Mel'nikov-Meshkov calculation to magnetization relaxation of single-domain ferromagnetic particles possessing nonaxially symmetric potentials of the magnetocrystalline anisotropy. (We remark that the magnetic relaxation problem differs fundamentally from that of mechanical particles because the undamped equation of motion of the magnetization of a single domain ferromagnetic particle is the gyromagnetic equation. Thus the inertia plays no role; the part played by inertia in the mechanical system is essentially mimicked in the magnetic system for nonaxially symmetric potentials by the gyromagnetic term which gives rise to the coupling or "entanglement" of the transverse and longitudinal modes.)

It is the purpose of this paper to demonstrate that the Mel'nikov and Meshkov approach applied to the particular problem of the inertial Brownian motion of a fixed axis rotator in a double well cosine potential yields an accurate solution for the greatest relaxation time $\lambda_{1}^{-1}$ for high barriers and for all values of the dissipation. Such a potential allows the flipping of rotators to neighboring wells, thus permitting both relaxation and oscillatory behavior in the same model. The detailed description of the model (in the VHD or noninertial limit) is given in Refs. 2 (Chap. 4), 22, and 23 in connection with site models of dielectric relaxation in molecular crystals and polar liquids. The first attempts to include inertial effects (which are of importance in the rotation in the VLD region) were made in Refs. 24-27. These calculations are, however, only valid in a restricted range of values of the dissipation parameter and it is only with the advent of the matrix continued fraction method ${ }^{28,29}$ that reliable results have become available for all values of the dissipation parameter, including the VLD region. ${ }^{1,2}$ Here, we calculate the Kramers escape rate by the Mel'nikov-Meshkov asymptotic (in the sense that it applies for high barriers) and universal (in the sense that it is valid for all values of the damping) formula and compare its inverse with the greatest relaxation time predicted by the exact matrix continued fraction solution of the underlying Langevin equation. In the following section, we shall briefly review the derivation of a universal formula for the Kramers escape rate as applied to Brownian rotation in a double well potential.

\section{UNIVERSAL FORMULA FOR THE ESCAPE RATE FOR FIXED AXIS ROTATION}

Our starting point is the Langevin equation for a dipole $\boldsymbol{\mu}$ rotating about an axis normal to the $x y$ plane, ${ }^{2}$

$$
I \ddot{\phi}(t)+\zeta \dot{\phi}(t)+\frac{d V(\phi)}{d \phi}=\lambda(t),
$$

where $I$ is the moment of inertia of a rotator about the axis of rotation, $\phi$ is the angle specifying the angular position of a rotator, $\zeta \dot{\phi}(t)$ and $\lambda(t)$ are the frictional and white-noise torques acting on a rotator due to the Brownian motion arising from the heat bath. The internal field due to molecular interactions is represented by the twofold cosine potential (see Fig. 2),

$$
V(\phi)=V_{0}[\cos (2 \phi)-1]=-2 k T \sigma \sin ^{2} \phi,
$$

where $2 \sigma=2 V_{0} / k T$ is the barrier height parameter. The corresponding Klein-Kramers (Fokker-Planck) equation for the joint probability density function $W(\phi, \dot{\phi}, t)$ of the angle $\phi$ and angular velocity $\dot{\phi}$ can be written as ${ }^{2}$

$$
\frac{\partial W}{\partial t}=L_{\mathrm{FP}} W,
$$




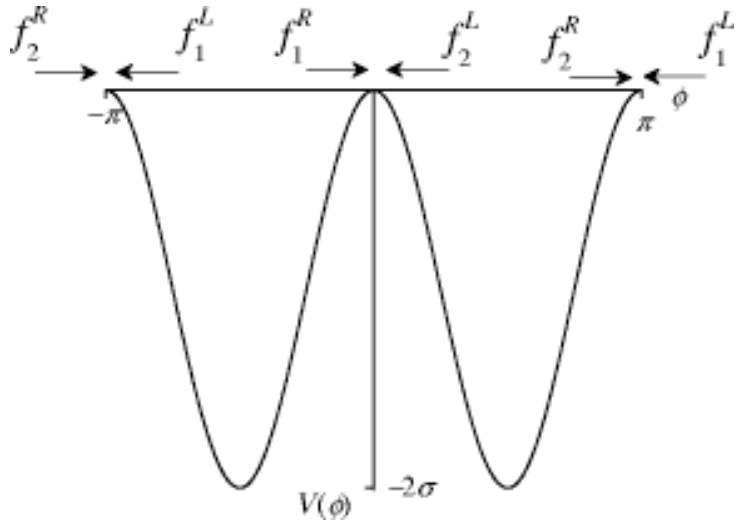

FIG. 2. Potential function $V(\phi)$ from Eq. (8).

where $\beta=\zeta / I$ and the Fokker-Planck operator $L_{\mathrm{FP}}$ is given by $^{27}$

$$
\begin{aligned}
L_{\mathrm{FP}} W= & -\dot{\phi} \frac{\partial W}{\partial \phi}+\frac{1}{I} \frac{d V}{d \phi} \frac{\partial W}{\partial \dot{\phi}} \\
& +\beta\left(\frac{\partial}{\partial \dot{\phi}}(\dot{\phi} W)+\frac{k T}{I} \frac{\partial^{2} W}{\partial \dot{\phi}^{2}}\right) .
\end{aligned}
$$

The first two terms on the right-hand side of Eq. (10) comprise the convective or Liouville term describing in the absence of dissipation the undamped streaming motion along the energy trajectories in phase space corresponding to Hamilton's equations. The last term (the diffusion term) represents the interchange of energy (dissipative coupling) with the heat bath.

Since escape over the potential barrier generated by Eq. (8) is exponentially slow for $\sigma \gg 1$ (high barriers), we may consider the quasistationary distribution $W(\phi, \dot{\phi})$ which does not depend explicitly on the time. Thus Eq. (9) can be rewritten as $L_{\mathrm{FP}} W=0$ or

$$
I \dot{\phi} \frac{\partial W}{\partial \phi}=\frac{d V(\phi)}{d \phi} \frac{\partial W}{\partial \dot{\phi}}+I \beta\left(\frac{\partial}{\partial \dot{\phi}}(\dot{\phi} W)+\frac{k T}{I} \frac{\partial^{2} W}{\partial \dot{\phi}^{2}}\right) .
$$

We shall now apply the Mel'nikov-Meshkov method to the potential given by Eq. (8). Since our problem differs in detail from the translational Brownian motion considered by them, we shall give a condensed version of the modifications required in order to apply their method to the present problem. First, we note that the particular Fokker-Planck equation given above may be represented in terms of angle-energy (fast and slow variable) coordinates $\{\phi, E\}$ [where $E$ $\left.=I \dot{\phi}^{2} / 2+V(\phi)\right]$ using the transformations ${ }^{7,9}$

$$
\begin{aligned}
& \frac{\partial}{\partial \dot{\phi}} f^{R, L}(\phi, p)= \pm \sqrt{2 I[E-V(\phi)]} \frac{\partial}{\partial E} f^{R, L}(\phi, E), \\
& \frac{\partial}{\partial \phi} f^{R, L}(\phi, p)=\frac{\partial}{\partial \phi} f^{R, L}(\phi, E)+\frac{d V(\phi)}{d \phi} \frac{\partial}{\partial E} f^{R, L}(\phi, E),
\end{aligned}
$$

where

$$
f^{R}(\phi, E)=W(\phi, \dot{\phi})=W(\phi, \sqrt{2[E-V(\phi)] / I}),
$$

$$
f^{L}(\phi, E)=W(\phi,-\dot{\phi})=W(\phi,-\sqrt{2[E-V(\phi)] / I}) .
$$

The function $f^{R}(\phi, E)$ gives the distribution of rotators rotating clockwise while the function $f^{L}(\phi, E)$ gives the distribution of rotators rotating counterclockwise. Thus we have from Eqs. (11)-(15)

$$
\frac{\partial f^{R, L}}{\partial \phi}= \pm \sqrt{2 I[E-V(\phi)]} \beta \frac{\partial}{\partial E}\left[f^{R, L}+k T \frac{\partial}{\partial E} f^{R, L}\right] .
$$

Furthermore, if we define the action $s(\phi)$ in the usual way as $^{30}$

$$
\frac{d s}{d \phi}= \pm \sqrt{2 I[E-V(\phi)]}
$$

Eq. (16) can compactly be rewritten as the energy-action diffusion equation ${ }^{7,9}$

$$
\frac{\partial f^{R, L}}{\partial s}=\beta \frac{\partial}{\partial E}\left[f^{R, L}+k T \frac{\partial}{\partial E} f^{R, L}\right] .
$$

We emphasize that the solution of Eq. (18) will effectively become Maxwell-Boltzmann distributions deep in the wells of the potential given by Eq. (8) and will differ from those distributions only in a relatively narrow region about the top of the barrier. The behavior being analogous to the VLD case, however, the angle (the fast variable) dependence of the function $f$ given by the left-hand side of Eq. (18) may not now be neglected near the top of the barrier (compare, for example, pp. 538 and 539 of Ref. 7). Equation (18) has a formal solution as the convolution, ${ }^{7,9}$

$$
\begin{aligned}
f^{R, L}(s, E)= & \int_{E} \int_{s} g^{R, L}\left(s-s^{\prime}, E-E^{\prime}\right) \\
& \times f^{R, L}\left(s^{\prime}, E^{\prime}\right) d s^{\prime} d E^{\prime},
\end{aligned}
$$

where the kernel $g(s, E)$ is the Green function given by ${ }^{7,9}$

$$
\begin{aligned}
& g\left(s, E-E^{\prime}\right)=\frac{1}{\sqrt{4 \pi k T \beta s}} \exp \left[-\frac{\left(E-E^{\prime}+\beta s\right)^{2}}{4 k T \beta s}\right], \\
& g\left(0, E-E^{\prime}\right)=\delta\left(E-E^{\prime}\right),
\end{aligned}
$$

and the limits of integration are determined by the boundary conditions for the functions $f^{R}(s, E)$ and $f^{L}(s, E)$. We proceed as follows. Near the barrier, the flux of the left-going particles inside the first well arises by reflection from the barrier of the right-going particles with $E<0$ (recall that $E$ $\simeq 0$ is the barrier energy) and also from the particles which have crossed over the barrier from the second well with $E$ $>0$ (see Fig. 2). The same conditions hold for the second well yielding the following relationship between $f_{1,2}^{R}$ and $f_{1,2}^{L}$ (subscripts 1 and 2 pertain to the wells):

$$
\begin{aligned}
& f_{1}^{L}\left[\phi^{\prime}(E), E\right]=f_{1}^{R}\left[\phi^{\prime}(E), E\right], \\
& f_{2}^{R}\left[\phi^{\prime \prime}(E), E\right]=f_{2}^{L}\left[\phi^{\prime \prime}(E), E\right], \quad(E<0),
\end{aligned}
$$




$$
f_{1}^{L}(0, E)=f_{2}^{L}(0, E), \quad f_{1}^{R}(0, E)=f_{2}^{R}(0, E), \quad(E>0),
$$

where $\phi^{\prime}(E)$ and $\phi^{\prime \prime}(E)$ are the roots of the equations $V_{1}\left(\phi^{\prime}\right)=E$ and $V_{2}\left(\phi^{\prime \prime}\right)=E$, respectively. Particles with different energies $E$ are always reflected at different angles $\phi$. For $E \sim k T$ (the order of magnitude of a fluctuation), however, this difference is small compared to the angular size of the potential well. Thus we may assume that such particles propagate along trajectories very close to the barrier energy trajectory (defined by $V_{1}=V_{2}=0$ ) and so can be described by identical Green functions (20), viz.,

$$
g_{S}(E)=g(S, E)=\frac{1}{\sqrt{4 \pi k T \beta S}} \exp \left[-\frac{(E+\beta S)^{2}}{4 k T \beta S}\right],
$$

where $S=S_{i}(i=1,2)$ and $S_{i}$ is the action in the $i$ th well given by

$$
\begin{aligned}
& S_{1}=\int_{-\pi}^{0} \sqrt{-2 I V(\phi)} d \phi=4 \sqrt{I k T \sigma} \\
& S_{2}=\int_{0}^{\pi} \sqrt{-2 I V(\phi)} d \phi=4 \sqrt{I k T \sigma} .
\end{aligned}
$$

The complete system of integral equations is then

$$
\begin{aligned}
f_{i}^{R}(E)= & \int_{-\infty}^{\infty}\left[g_{S}\left(E-E^{\prime}\right) f_{i}^{L}\left(E^{\prime}\right) \theta\left(-E^{\prime}\right)\right. \\
& \left.+g_{S}\left(E-E^{\prime}\right) f_{j}^{R}\left(E^{\prime}\right) \theta\left(E^{\prime}\right)\right] d E^{\prime}, \\
f_{i}^{L}(E)= & \int_{-\infty}^{\infty}\left[g_{S}\left(E-E^{\prime}\right) f_{i}^{R}\left(E^{\prime}\right) \theta\left(-E^{\prime}\right)\right. \\
& \left.+g_{S}\left(E-E^{\prime}\right) f_{j}^{L}\left(E^{\prime}\right) \theta\left(E^{\prime}\right)\right] d E^{\prime}
\end{aligned}
$$

$(i, j=1,2$ and $i \neq j)$, where $\theta(x)$ is the Heaviside unit step function. The system of four integral equations (23) and (24) can be reduced to

$$
\begin{aligned}
f_{1}(E)= & \int_{-\infty}^{\infty}\left[g_{S}\left(E-E^{\prime}\right) f_{1}\left(E^{\prime}\right) \theta\left(-E^{\prime}\right)\right. \\
& \left.+g_{S}\left(E-E^{\prime}\right) f_{2}\left(E^{\prime}\right) \theta\left(E^{\prime}\right)\right] d E^{\prime}, \\
f_{2}(E)= & \int_{-\infty}^{\infty}\left[g_{S}\left(E-E^{\prime}\right) f_{2}\left(E^{\prime}\right) \theta\left(-E^{\prime}\right)\right. \\
& \left.+g_{S}\left(E-E^{\prime}\right) f_{1}\left(E^{\prime}\right) \theta\left(E^{\prime}\right)\right] d E^{\prime},
\end{aligned}
$$

where $f_{1}(E)=f_{1}^{L}(E)+f_{1}^{R}(E)$ and $f_{2}(E)=f_{2}^{L}(E)+f_{2}^{R}(E)$. Equations (25) and (26) indicate that two distinct contributions to $f_{1}$ and $f_{2}$ exist. One contribution is from particles reflected from the barrier with distribution $f_{1} \theta(-E)$ and $f_{2} \theta(-E)$ and the other is from particles which have crossed the barrier with distribution $f_{2} \theta(E)$ and $f_{1} \theta(E)$.

Now, the escape rate $\Gamma_{12}$ from the well 1 is (see Fig. 2)

$$
\begin{aligned}
\Gamma_{12} & =\int_{0}^{\infty}\left[f_{1}^{L}(E)+f_{1}^{R}(E)-f_{2}^{L}(E)-f_{2}^{R}(E)\right] d E \\
& =\int_{0}^{\infty}\left[f_{1}(E)-f_{2}(E)\right] d E .
\end{aligned}
$$

Due to the equivalence of the wells 1 and 2, the escape rate $\Gamma_{21}$ from the well 2 is equal to $\Gamma_{12}$. These escape rates are related to the lifetime $\tau$ of a particle by

$$
\tau^{-1}=\Gamma_{12}+\Gamma_{21}=2 \Gamma_{12}=2 \int_{0}^{\infty}\left[f_{1}(E)-f_{2}(E)\right] d E .
$$

Solving Eqs. (23) and (25) by the Wiener-Hopf method, ${ }^{9}$ as shown in Appendix A, and using the solution so obtained to evaluate of $\tau^{-1}$ from Eq. (28), we have

$$
\begin{aligned}
\tau^{-1}= & \frac{A^{\prime 2}(\Delta)}{\pi \eta A^{\prime}(2 \Delta)}\left[\sqrt{\frac{\beta^{\prime 2}}{4}+\eta^{2}\left|V^{\prime \prime}(0) / I\right|}-\frac{\beta^{\prime}}{2}\right] \\
& \times\left[\sqrt{\frac{V^{\prime \prime}\left(\phi_{1}\right)}{\left|V^{\prime \prime}(0)\right|}} e^{V\left(\phi_{1}\right) /(k T)}\right. \\
& \left.+\sqrt{\frac{V^{\prime \prime}\left(\phi_{2}\right)}{\left|V^{\prime \prime}(0)\right|}} e^{V\left(\phi_{2}\right) /(k T)}\right],
\end{aligned}
$$

where $\eta=\sqrt{I / 2 k T}$ is a characteristic time, $\beta^{\prime}=\beta \eta$ is the dimensionless damping parameter,

$$
V\left(\phi_{i}\right) /(k T)=-2 \sigma, \quad\left|V^{\prime \prime}\left(\phi_{i}\right)\right| / I=\left|V^{\prime \prime}(0)\right| / I=2 \sigma / \eta^{2},
$$
$\phi_{i}=\phi_{\min _{i}}(i=1,2)$ are the potential minima in the $i$ th well,

$$
\begin{aligned}
& A^{\prime}(\Delta)=\exp \left[\frac{1}{\pi} \int_{0}^{\infty} \frac{\ln \left[1-\exp \left\{-\Delta\left(\lambda^{2}+1 / 4\right)\right\}\right]}{\lambda^{2}+1 / 4} d \lambda\right], \\
& \Delta=\frac{\beta^{\prime} S}{\eta k T}=4 \beta^{\prime} \sqrt{2 \sigma} .
\end{aligned}
$$

As shown by Mel'nikov and Meshkov, ${ }^{9}$

$$
A^{\prime}(\Delta) \rightarrow 1 \text { as } \Delta \rightarrow \infty \quad \text { and } A^{\prime}(\Delta) / \Delta \rightarrow 1 \text { as } \Delta \rightarrow 0 .
$$

Thus the greatest relaxation time $\tau$ can now be compactly represented by the universal formula

$$
\tau=\frac{A^{\prime}(2 \Delta)}{A^{\prime 2}(\Delta)} \tau_{\mathrm{IHD}},
$$

where

$$
\tau_{\mathrm{IHD}}=\frac{\pi \eta}{\sqrt{\beta^{\prime 2}+8 \sigma}-\beta^{\prime}} e^{2 \sigma}
$$

is the greatest relaxation time in the IHD limit. The leading factor on the right-hand side of Eq. (33) is the correction to the IHD result due to Mel'nikov and Meshkov. If $\beta^{\prime} \rightarrow \infty$, we have from Eqs. (32) and (33) the VHD formula [cf. Ref. 2, Sec. 4.5]

$$
\tau_{\mathrm{VHD}}=\frac{\pi \eta \beta^{\prime}}{4 \sigma} e^{2 \sigma},
$$

which is the result of Lauritzen and Zwanzig ${ }^{22}$ (in our notation). In like manner, in the VLD limit, $\beta^{\prime} \rightarrow 0$, we have

$$
\tau_{\mathrm{VLD}}=\frac{\pi \eta}{8 \beta^{\prime} \sigma} e^{2 \sigma}
$$


Since the potential is symmetric the greatest relaxation time $\tau$, Eq. (33), can be used to estimate the correlation time $\tau_{\|}$of the equilibrium correlation function $C(t)$ of the longitudinal component of the dipole moment which for the potential given by Eq. (8) is

$$
C(t)=\frac{\langle\sin \phi(0) \sin \phi(t)\rangle_{0}}{\left\langle\sin ^{2} \phi(0)\right\rangle_{0}}
$$

(the angular brackets denote the equilibrium ensemble average). The correlation time $\tau_{\|}$is defined as the area under the curve of $C(t)$, viz.,

$$
\tau_{\|}=\int_{0}^{\infty} C(t) d t
$$

The time $\tau_{\|}$may equivalently be defined in terms of the eigenvalues $\left(\lambda_{k}\right)$ of the Fokker-Planck operator $L_{\mathrm{FP}}$ from Eq. (10) because the function $C(t)$ may be formally written as the discrete set of relaxation modes

$$
C(t)=\sum_{k} c_{k} e^{-\lambda_{k} t} .
$$

Thus from Eqs. (38) and (39):

$$
\tau_{\|}=\sum_{k} c_{k} / \lambda_{k},
$$

where $\Sigma_{k} c_{k}=1$. The correlation time $\tau_{\|}$contains contributions from all the eigenvalues $\lambda_{k}$. In general, in order to evaluate $C(t)$ and $\tau_{\|}$, a knowledge of all the $\lambda_{k}$ and $c_{k}$ is required. However, in the high barrier limit $(\sigma \gg 1), \lambda_{1}$ $\sim e^{-2 \sigma \ll \lambda_{k}}$ and for symmetric potentials $c_{1} \approx 1 \gg c_{k}(k$ $\neq 1),{ }^{1,2}$ so that the approximation $\tau_{\|} \approx 1 / \lambda_{1}$ can be used. In other words, the inverse of the smallest nonvanishing eigenvalue, i.e., the greatest relaxation time closely approximates the correlation time $\tau_{\|}$for symmetric potentials in the lowtemperature (high barrier) limit.

\section{EXACT MATRIX CONTINUED FRACTION SOLUTION OF THE LANGEVIN EQ. (7)}

In order to calculate $\tau_{\|}$, we shall use the matrix continued fraction approach, developed for a similar problem in Ref. 29. This is accomplished as follows. As shown in Ref. 2 (Chaps. 4 and 10), we can derive from the Langevin Eq. (7) the following recurrence relation for the correlation functions $c_{n, q}(t)$, viz.,

$$
\begin{gathered}
\eta \dot{c}_{n, q}(t)+n \beta^{\prime} c_{n, q}(t)+\frac{i q}{2}\left[c_{n+1, q}(t)+2 n c_{n-1, q}(t)\right] \\
-i n \sigma\left[c_{n-1, q+2}(t)-c_{n-1, q-2}(t)\right]=0,
\end{gathered}
$$

where

$$
\begin{aligned}
c_{n, q}(t)= & \left\langle\sin \phi(0) H_{n}[\eta \dot{\phi}(t)] e^{-i q \phi(t)}\right\rangle, \\
& (n \geqslant 0,-\infty<q<\infty),
\end{aligned}
$$

so that $\operatorname{Im}\left[c_{0,1}(t)\right] / \operatorname{Im}\left[c_{0,1}(0)\right]=C(t)$, and $H_{n}$ are the Hermite polynomials. ${ }^{31}$ By Laplace transformation, we have from Eq. (41)

$$
\begin{aligned}
& {\left[\eta s+n \beta^{\prime}\right] \tilde{c}_{n, q}(s)+\frac{i q}{2}\left[\tilde{c}_{n+1, q}(s)+2 n \tilde{c}_{n-1, q}(s)\right]} \\
& \quad-i n \sigma\left[\tilde{c}_{n-1, q+2}(s)-\tilde{c}_{n-1, q-2}(s)\right]=\eta c_{0, q}(0) \delta_{n, 0} .
\end{aligned}
$$

Here we have noted that the initial conditions for $c_{n, q}(0)$ are

$$
\begin{aligned}
c_{0,2 q}(0)= & , \\
c_{0,2 q+1}(0) & =\left\langle\sin \phi e^{-i(2 q+1) \phi}\right\rangle \\
& =\frac{\int_{0}^{2 \pi} \sin \phi e^{-i(2 q+1) \phi} e^{-\sigma \cos 2 \phi} d \phi}{\int_{0}^{2 \pi} e^{-\sigma \cos 2 \phi} d \phi} \\
& =i(-1)^{q+1} \frac{I_{q}(\sigma)+I_{q+1}(\sigma)}{2 I_{0}(\sigma)},
\end{aligned}
$$

where the $I_{n}$ are the modified Bessel functions of the first kind of order $n ;{ }^{31}$ the other $c_{n, q}(0)=0$ for $n \geqslant 1$ because

$$
\left\langle\sin \phi H_{n}(\dot{\phi}) e^{-i q \phi}\right\rangle=0
$$

for the equilibrium Maxwell-Boltzmann distribution.

In order to solve Eq. (43), we introduce the column vectors

$$
\widetilde{\mathbf{C}}_{1}(s)=\left(\begin{array}{c}
\vdots \\
\tilde{c}_{0,-2}(s) \\
\tilde{c}_{0,-1}(s) \\
\widetilde{c}_{0,1}(s) \\
\widetilde{c}_{0,2}(s) \\
\vdots
\end{array}\right)
$$

and

$$
\widetilde{\mathbf{C}}_{n}(s)=\left(\begin{array}{c}
\vdots \\
\widetilde{c}_{n-1,-2}(s) \\
\widetilde{c}_{n-1,-1}(s) \\
\widetilde{c}_{n-1,0}(s) \\
\widetilde{c}_{n-1,1}(s) \\
\tilde{c}_{n-1,2}(s) \\
\vdots
\end{array}\right) \quad(n \geqslant 2) .
$$

Now, Eq. (43) can be rearranged as the set of matrix threeterm recurrence equations,

$$
\begin{aligned}
{[\eta s} & \left.+\beta^{\prime}(n-1)\right] \widetilde{\mathbf{C}}_{n}(s)-\mathbf{Q}_{n}^{+} \widetilde{\mathbf{C}}_{n+1}(s)-\mathbf{Q}_{n}^{-} \widetilde{\mathbf{C}}_{n-1}(s) \\
& =\eta \delta_{n, 1} \mathbf{C}_{1}(0), \quad(n \geqslant 1),
\end{aligned}
$$

where the column vector $\mathbf{C}_{1}(0)$ and the matrices $\mathbf{Q}_{n}^{+}$and $\mathbf{Q}_{n}^{-}$ are given in Appendix B. By invoking the general method for solving the matrix recurrence Eq. (44), ${ }^{2}$ we have the exact solution for the spectrum $\widetilde{\mathbf{C}}_{1}(s)$ in terms of a matrix continued fraction, viz.,

$$
\widetilde{\mathbf{C}}_{1}(s)=\eta \Delta_{1}(s) \mathbf{C}_{1}(0),
$$

where the matrix continued fraction $\Delta_{n}(s)$ is defined by the recurrence equation

$$
\Delta_{n}(s)=\left\{\left[\eta s+\beta^{\prime}(n-1)\right] \mathbf{I}-\mathbf{Q}_{n}^{+} \Delta_{n+1}(s) \mathbf{Q}_{n+1}^{-}\right\}^{-1} .
$$

and $\mathbf{I}$ is the unit matrix. Having determined $\widetilde{\mathbf{C}}_{1}(s)$, we can evaluate the correlation time $\tau_{\|}$, 

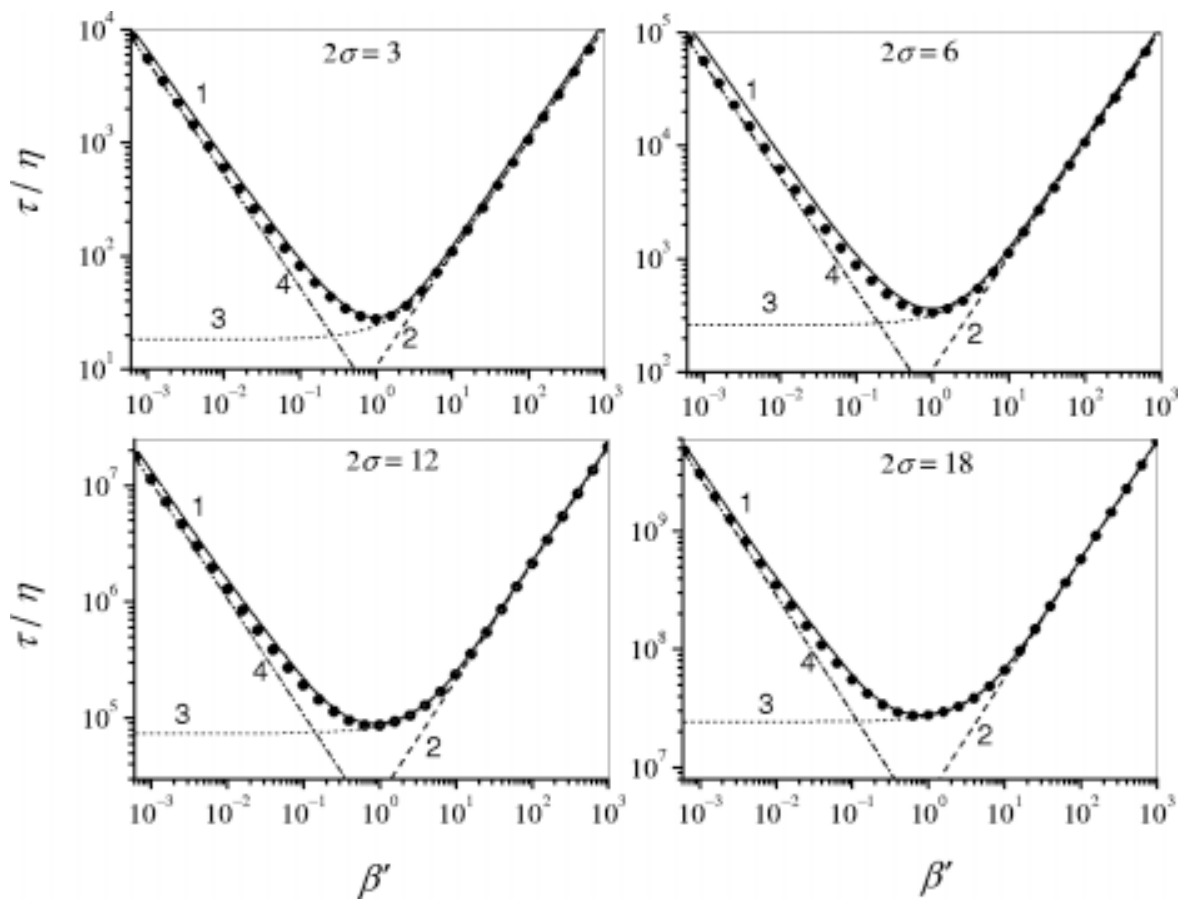

FIG. 3. $\pi / \eta$ vs $\beta^{\prime}$ for barrier height parameters $2 \sigma=3,6,12$, and 18 . Solid lines 1: exact matrix continued fraction solution for the correlation time $\tau_{\|}$, Eq. (46); dashed lines 2: the VHD Eq. (35); dotted line 3: the IHD Eq. (34); dashed-dotted lines 4: the VLD Eq. (36); filled circles: the universal Mel'nikov-Meshkov Eq. (33).

$$
\tau_{\|}=\tilde{C}(0)=\frac{\tilde{c}_{0,-1}(0)-\tilde{c}_{0,1}(0)}{c_{0,-1}(0)-c_{0,1}(0)},
$$

as well as the spectrum of the longitudinal correlation function $\widetilde{C}(\omega)$,

$$
\tilde{C}(\omega)=\frac{\tilde{c}_{0,-1}(i \omega)-\tilde{c}_{0,1}(i \omega)}{c_{0,-1}(0)-c_{0,1}(0)} .
$$

We remark that for free Brownian rotation of plane rotators $(\sigma=0)$, the exact analytic solution for $\widetilde{C}(\omega)$ may be expressed $^{32}$ in terms of the confluent hypergeometric (Kummer's) function $M(a, b, z)^{31}$ (in our notation)

$$
\begin{aligned}
\widetilde{C}(\omega)= & \frac{2 \beta^{\prime} \eta}{1+i \omega 2 \beta^{\prime} \eta} \\
& \times M\left[1,1+2 \beta^{\prime-2}\left(1+i \omega 2 \beta^{\prime} \eta\right), 2 \beta^{\prime-2}\right] .
\end{aligned}
$$

In the VLD and VHD limits, the correlation time $\tau_{\|}=\tilde{C}(0)$ from Eq. (48) yields

$$
\tau_{\|} \rightarrow \eta \sqrt{\pi} \quad \text { and } \tau_{\|} \rightarrow 2 \eta \beta^{\prime},
$$

respectively. Equations (48) and (49) provide very useful relations for the purpose of testing the results of numerical calculations.

\section{RESULTS AND DISCUSSION}

The exact matrix continued fraction solution [Eq. (44)] we have obtained is easily computed (algorithms for calculating matrix continued fractions are discussed in Refs. 1 and 2). As far as practical calculations of the infinite matrix continued fraction are concerned, we approximate it by a matrix continued fraction of finite order (by putting $\boldsymbol{\Delta}_{n+1}=\mathbf{0}$ at some $n=N)$. Simultaneously, we confine the dimensions of the infinite matrices $\mathbf{Q}_{n}^{-}, \mathbf{Q}_{n}^{+}$, and $\mathbf{I}$ to a finite value $M$ $\times M$. Both $N$ and $M$ depend on the dimensionless barrier $(\sigma)$ and damping $\left(\beta^{\prime}\right)$ parameters and must be chosen taking into account the desired degree of accuracy of the calculation. Both $N$ and $M$ increase with decreasing $\beta^{\prime}$ and increasing $\sigma$. For example, for $\sigma=6$ (a relatively high barrier $=12 \mathrm{kT}$ ), $N$ $=50$ and $M=60$ allows us to obtain six significant digits for $\tau / \eta=8.88490 \times 10^{4}$ at $\beta^{\prime}=1$ (intermediate damping), while $N=2300$ and $M=300$ are required to obtain six significant digits for $\tau / \eta=1.47885 \times 10^{6}$ at $\beta^{\prime}=0.01$ (low damping).

The greatest relaxation time $\tau$ predicted by the Mel'nikov and Meshkov ${ }^{9}$ method [Eq. (33)] and the correlation time $\tau_{\|}$calculated numerically by matrix continued fraction methods from Eqs. (45) and (46) are shown in Figs. 3 and 4 as functions of $\beta^{\prime}$ and $\sigma$, respectively. Here, the VHD [Eq. (35)], IHD [Eq. (34)], and VLD [Eq. (36)] asymptotes are also shown for comparison. Apparently in the high barrier limit, Eq. (33) provides a good approximation of the correlation time $\tau_{\|}$for all values of the damping parameter $\beta^{\prime}$ including the VHD, VLD, and crossover regions. Furthermore, Eq. (33) yields a reasonable estimate for $\tau_{\|}$even for low barriers, $\sigma \sim 1$ (see Fig. 4). However, a marked difference (of order of $10-40 \%$ ) between numerical and analytical results exists in the VLD region especially at moderate barriers (this difference decreases with increasing $\sigma$ and decreasing $\beta^{\prime}$, see Fig. 3). Such behavior has already been noted for other systems (see, e.g., Refs. 14 and 16). Thus in order to improve the accuracy of the universal turnover formula, Melnikov ${ }^{33}$ suggested a systematic way of accounting for finite-barrier corrections. Analysis of the translational Brownian motion in a cosine potential demonstrates that if such corrections are included, the accuracy of the universal formula is considerably improved. ${ }^{34,35}$ His method may also be applied to the present problem.

We remark that in principle the accurate calculation of the correlation time $\tau_{\|}$is a much more complicated problem than the evaluation of the smallest nonvanishing eigenvalue 

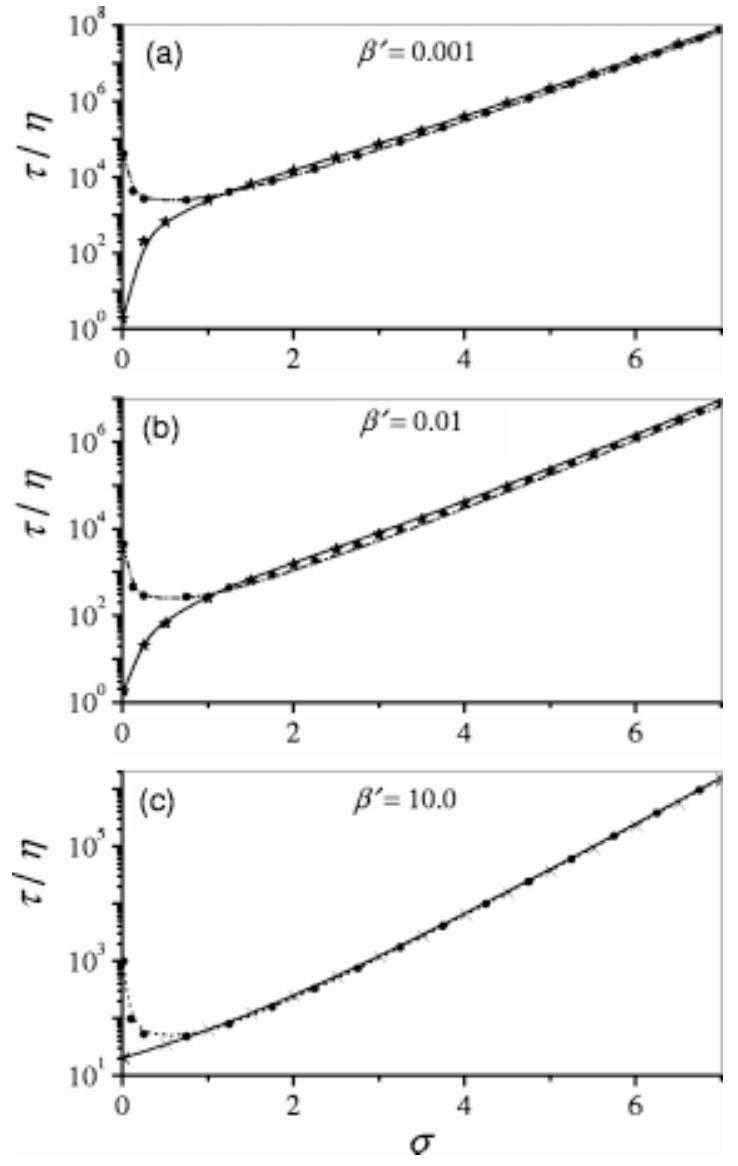

FIG. 4. $\tau / \eta$ vs $\sigma$ for $\beta^{\prime}=0.001$ [(a) very low damping, $\beta^{\prime}=0.01$ (b) low damping, and $\beta^{\prime}=10$ (c) high damping]. Solid lines: exact matrix continued fraction solution for the correlation time $\tau_{\|}$, Eq. (46), dashed-dotted lines: the VLD Eq. (36); dashed lines: the IHD Eq. (35) for $\beta^{\prime}=10$; filled circles: the universal Mel'nikov-Meshkov Eq. (33): crosses: the VHD Eq. (52); stars: the VLD Eq. (56).

alone since all the other eigenvalues give a contribution to $\tau_{\|}$ (see Sec. II). Fortunately, for the problem under consideration, an accurate method of estimating $\tau_{\|}$in the VHD and VLD limits exists. This method based on the mean first passage time was first suggested by Szabo $^{36}$ in the context of the theory of polarized fluorescent emission in uniaxial liquid crystals. However, it may be used for all systems with dynamics governed by single variable Fokker-Planck equations. Namely, one may calculate in intergral form the correlation time $\tau_{A}$ of a dynamic variable $A(x)$ defined as the area under the curve of the normalized autocorrelation function $C_{A}(t)=\langle A[x(0)] A[x(t)]\rangle_{0}$. Here \langle\rangle$_{0}$ designates the statistical averages over the stationary (equilibrium) distribution function $W_{\mathrm{st}}[x(0)]$ with $x(0)$ defined in the range $x_{1}$ $\leqslant x(0) \leqslant x_{2}$ and it is assumed that $\langle A\rangle_{0}=0$. The pertinent feature of these is that an exact formula for the correlation time $\tau_{A}$, may be expressed in terms of the diffusion coefficient $D^{(2)}(x)$ and $W_{\text {st }}(x)$ only (see, e.g., Ref. 1, Sec. S.9, and Ref. 2, Chap. 2, Sec. 2.10, for details), viz.,

$$
\tau_{A}=\frac{1}{\left\langle A^{2}\right\rangle_{0}} \int_{x_{1}}^{x_{2}} \frac{1}{W_{\mathrm{st}}(x) D^{(2)}(x)}\left[\int_{x_{1}}^{x} A(z) W_{\mathrm{st}}(z) d z\right]^{2} d x .
$$

The advantage of Eq. (50) is that it yields VHD and VLD asymptotes, valid for all barrier heights including very low barriers, where the Melnikov-Meshkov method is not applicable.

Since the dynamics of the system of planar rotators in the VHD and VLD limits are governed by a single variable, we can obtain accurate VHD and VLD asymptotes by applying Eq. (50) to the present problem. In the high damping limit $\left(\beta^{\prime} \gg 1\right)$, the appropriate single variable Fokker-Planck (Smoluchowski) equation for the probability density function $W(\phi, t)$ of the orientations of rotators is ${ }^{2,22}$

$$
\frac{\partial}{\partial t} W(\phi, t)=\frac{1}{2 \beta^{\prime} \eta} \frac{\partial}{\partial \phi}\left(\frac{\partial}{\partial \phi}-2 \sigma \sin 2 \phi\right) W(\phi, t) .
$$

Thus, noting that $D^{(2)}(\phi)=1 /\left(2 \beta^{\prime} \eta\right)$ and $A=\sin \phi$, the correlation time $\tau_{\|}$of the longitudinal dipole moment autocorrelation function $C(t)=\langle\sin \phi(0) \sin \phi(t)\rangle_{0}$ is given by

$$
\begin{aligned}
\tau_{\|} \sim \tau_{\|}^{\mathrm{VHD}} & =\frac{2 \beta^{\prime} \eta}{\left\langle\sin ^{2} \phi\right\rangle_{0}} \int_{-\pi}^{\pi} \frac{1}{W_{\mathrm{st}}(\phi)}\left(\int_{0}^{\phi} \sin x W_{\mathrm{st}}(x) d x\right)^{2} d \phi \\
& =\frac{\beta^{\prime} \eta e^{2 \sigma}}{\sigma\left[I_{1}(\sigma)+I_{0}(\sigma)\right]} \int_{0}^{\pi / 2} e^{\sigma \cos 2 \phi} \operatorname{erf}^{2}(\sqrt{2 \sigma} \cos \phi) d \phi .
\end{aligned}
$$

Here $W_{\mathrm{st}}(\phi)=e^{-\sigma+2 \sigma \sin ^{2} \phi} /\left[2 \pi I_{0}(\sigma)\right]$ is the equilibrium Boltzmann distribution function [which is a stationary solution of Eq. (51)], $\operatorname{erf}(z)$ is the error function, ${ }^{31}$ and we have noted that

$$
\left\langle\sin ^{2} \phi\right\rangle_{0}=\frac{I_{0}(\sigma)+I_{1}(\sigma)}{2 I_{0}(\sigma)} .
$$

In the opposite low damping limit $\left(\beta^{\prime} \ll 1\right)$, one may in order to obtain a single variable Fokker-Planck equation introduce the energy of the dipole

$$
\varepsilon=\eta^{2} \dot{\phi}^{2}-2 \sigma \sin ^{2} \phi
$$

and the time $w$ (phase) measured along a closed trajectory in phase space as action-angle variables. ${ }^{26}$ The energy $\varepsilon$ varies very slowly with time. Consequently, it is a slow variable in comparison to the phase $w$. By averaging the Fokker-Planck equation (9) over the fast phase variable $w$, Praestgaard and van Kampen ${ }^{26}$ derived a single variable Fokker-Planck equation for the probability density function $W(\varepsilon, t)$ in energy space (in our notation),

$$
\begin{aligned}
\frac{\partial}{\partial t} W(\varepsilon, t)= & \frac{2 \beta^{\prime}}{\eta}\left[\frac{\partial}{\partial \varepsilon}\left(\eta^{2} \overline{\overline{\dot{\phi}^{2}}}(\varepsilon)-\frac{1}{2}\right)\right. \\
& \left.+\eta^{2} \frac{\partial^{2}}{\partial \varepsilon^{2}} \overline{\dot{\phi}^{2}}(\varepsilon)\right] W(\varepsilon, t),
\end{aligned}
$$

where the double overbar denotes averaging over the fast phase variable. Since

$$
D^{(2)}(\varepsilon)=2 \beta^{\prime} \eta \overline{\overline{\dot{\phi}^{2}}}(\varepsilon)=\frac{2 \beta^{\prime}}{\eta}\left(\varepsilon+2 \sigma \overline{\overline{\sin ^{2}}} \phi(\varepsilon)\right),
$$


the correlation time $\tau_{\|}$is given by

$$
\begin{aligned}
\tau_{\|} \sim \tau_{\|}^{\mathrm{VLD}} & \frac{\eta}{2 \beta^{\prime}\left\langle\sin ^{2} \phi\right\rangle_{0}} \int_{-2 \sigma\left[\varepsilon+2 \sigma \sin ^{2} \phi(\varepsilon)\right] W_{\mathrm{st}}(\varepsilon)}^{\infty} \\
& \quad \times\left[\int_{-2 \sigma}^{\varepsilon} \overline{\overline{\sin \phi(x)}} W_{\mathrm{st}}(x) d x\right]^{2} d \varepsilon+\eta \sqrt{\pi} \\
\approx & \frac{\eta \sqrt{\pi} e^{\sigma}}{\beta^{\prime} \sqrt{2 \sigma}\left[I_{0}(\sigma)+I_{1}(\sigma)\right]} \int_{0}^{1} \frac{\cosh (2 \sigma m)-1}{(m-1) K(m)+E(m)} d m \\
& +\eta \sqrt{\pi},
\end{aligned}
$$

where $K(m)$ and $E(m)$ are complete elliptic integrals of the first and second kind, respectively. ${ }^{30}$ The calculation of the integrals in Eq. (56) is described in Appendix $\mathrm{C}$ [here we have used Eqs. (C7) $-(\mathrm{C} 11)]$. The term $\eta \sqrt{\pi}$ in Eq. (56) is due to the contribution of the free rotation to the correlation time (it is independent of $\beta^{\prime}$, and may be obtained from the solution of the undamped equation $\dot{W}=0$, see also Appendix C).

The regions of applicability of the VHD and VLD asymptotes from Eqs. (52) and (56) are the same as for the corresponding Fokker-Planck equations (51) and (55), viz., the VHD and VLD regions, respectively; in practice, Eqs. (52) and (56) may be used at $\beta^{\prime}>5$ and $\beta^{\prime}<0.01$. These VHD and VLD asymptotes are shown in Fig. 4. Apparently, these asymptotes yield a much better estimate for the correlation time than those provided by the Mel'nikov-Meshkov ${ }^{9}$ method (the maximum relative deviation between the corresponding curves is less then $25 \%$ in the worst cases, usually at the limits of applicability $\beta^{\prime} \sim 5$ [for Eq. (52)] and $\beta^{\prime} \sim 0.01$ [for Eq. (56); that is shown in Fig. 4].) In Fig. 5, we compare Eqs. (52) and (56) with the exact numerical solution for the correlation time at small barriers, where the Melnikov-Meshkov universal formula is not applicable. Here a simple ad hoc extrapolating equation ${ }^{6}$

$$
\tau_{\|} \sim \tau_{\|}^{\mathrm{VLD}}+\tau_{\|}^{\mathrm{VHD}}
$$

provides a satisfactory estimate of the correlation time $\tau_{\|}$for all damping. We emphasize that Eqs. (52), (56), and (57) can be used for all barrier heights $\sigma$ (see Fig. 4).

Mel'nikov ${ }^{8}$ and Pollak and co-workers ${ }^{12,17}$ have also extended the bridging integral method to take into account quantum effects. They attempt to generalize the classical formulas given by Kramers for the various escape rate regimes and the bridging integral Eq. (30) by incorporating in their integral equation for the energy distribution function the quantum tunneling factor for a parabolic barrier. We remark that the subject of tunneling in the context of the Kramers escape rate for rotational diffusion problems is of particular relevance in superparamagnetism. ${ }^{2}$ Here the Kramers theory as adapted to nonseparable Hamiltonians has been extensively used ${ }^{2,11}$ to study the reversal of the magnetization in single domain ferromagnetic particles. Here the magnetization may be considered as a macroscopic object since $10^{4}-10^{5}$ spins are collectively involved. A very important question first posed by Bean and Livingston ${ }^{37}$ is: does rever-
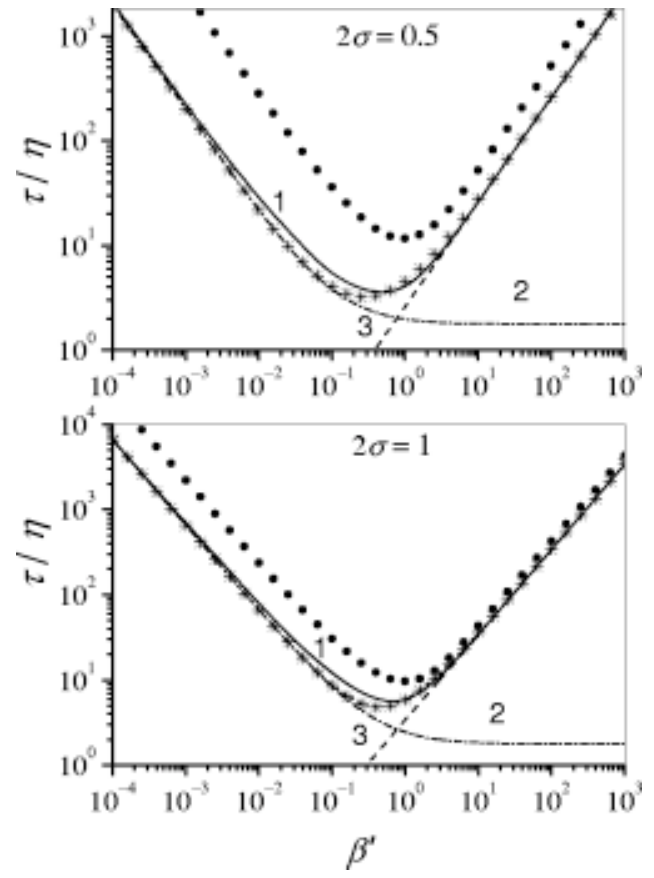

FIG. 5. $\tau / \eta$ vs $\beta^{\prime}$ for small barrier heights $2 \sigma=0.5$ and 1 . Solid lines 1 : exact matrix continued fraction solution for the correlation time $\tau_{\|}$, Eq. (46), dashed-dotted lines 2: the VLD Eq. (56); dashed lines 3: the VHD Eq. (52); filled circles: the universal Mel'nikov-Meshkov Eq. (33); stars: Eq. (57).

sal of magnetization by tunneling occur in such particles? If this reversal mechanism occurs then one would have an important example of macroscopic quantum tunneling. It follows therefore that the development of an accurate analytical formula for the Kramers escape rate incorporating tunneling effects is vital to the study of magnetization reversal mechanisms in superparamagnets and the possible existence of the macroscopic tunneling phenomenon in such systems. In the context of the present problem, we remark that the calculations of Mel'nikov and Meshkov may be confirmed as an accurate approximation to the exact escape rate because of the existence of the Klein-Kramers equation describing the evolution of the distribution function in phase space. In the context of single domain ferromagnetic particles, the corresponding evolution equation is Brown's Fokker-Planck equation for the distribution of the orientations of the magnetic moments on the unit sphere. Thus, in order to verify formulas for the Kramers escape rate which incorporate tunneling effects, it is necessary to define the quantummechanical master equation which underlies the relaxation process and to solve it numerically. It is possible that one may achieve this by using the Wigner representation of the quantum-mechanical master equation ${ }^{38-41}$ which in the classical limit goes over into the Klein-Kramers equation or Brown's Fokker-Planck equation. This representation of the quantum-mechanical problem lends itself to solution by the continued fraction method we have described.

Finally, we remark that Langer ${ }^{42}$ has generalized the Kramers IHD calculation to a system of many degrees of freedom and to non separable Hamiltonians yielding a general theory of the decay of metastable states. This calculation which may be successfully applied to magnetic relaxation is 
the generalization of Becker and Döring's ${ }^{43}$ calculation of the rate of condensation of a supersaturated vapor. The Mel'nikov and Meshkov method may also be applied to Langer's problem in order to extend his calculation to all values of the dissipation. However, one may well encounter difficulties in the evaluation of the action integrals which are involved in their integral formula in this general case.

\section{ACKNOWLEDGMENTS}

The support of this work by the CNRS Enterprise Ireland-France award scheme 2003 (program "Ulysses"), the Trinity College Dublin Trust, and INTAS (Project No. 01-2341) is gratefully acknowledged. We thank Professor D. S. F. Crothers for helpful conversations and Professor J.-L. Déjardin for useful comments.

\section{APPENDIX A: WIENER-HOPF METHOD OF SOLUTION OF EQS. (23) AND (25)}

Following Refs. 8 and 9, we can solve the integral Eqs. (23) and (25) by the Wiener-Hopf method by introducing the Fourier transforms ${ }^{9,44}$

$$
\varphi_{i}^{ \pm}(\lambda)=\tau_{\mathrm{TST}}\left(\phi_{i}\right) \int_{-\infty}^{\infty} f_{i}(E) \theta( \pm E) e^{-(i \lambda+1 / 2) E /(k T)} d E,
$$

where

$$
\tau_{\mathrm{TST}}^{-1}\left(\phi_{i}\right)=\frac{\sqrt{V^{\prime \prime}\left(\phi_{i}\right) / I}}{2 \pi} e^{V\left(\phi_{i}\right) /(k T)}
$$

and $\phi_{i}=\phi_{\min _{i}}$ are the potential minima in the $i$ th well [recalling that $V\left(\phi_{i}\right)$ is negative, see Fig. 2]. The prefactor $\tau_{\mathrm{TST}}\left(\phi_{i}\right)$ of the Fourier transformation which is introduced for notational convenience is suggested by Eq. (1). Applying this transformation to the integral Eqs. (23) and (25) we have

$$
\begin{aligned}
& \varphi_{1}^{+}(\lambda)+\varphi_{1}^{-}(\lambda)=\left[1-G_{1}(\lambda)\right]\left[\varphi_{1}^{-}(\lambda)+\varphi_{2}^{+}(\lambda)\right], \\
& \varphi_{2}^{+}(\lambda)+\varphi_{2}^{-}(\lambda)=\left[1-G_{2}(\lambda)\right]\left[\varphi_{2}^{-}(\lambda)+\varphi_{1}^{+}(\lambda)\right],
\end{aligned}
$$

where $G_{i}(\lambda)$ is related to the Fourier transform of the Green function $\widetilde{g}_{i}(\lambda)$ via

$$
G_{i}(\lambda)=1-\widetilde{g}_{i}(\lambda)=\exp \left[-\frac{\beta S_{i}}{k T}\left(\lambda^{2}+\frac{1}{4}\right)\right] .
$$

Now, Eq. (28) in turn can be represented as

$$
\begin{aligned}
\tau^{-1}= & \frac{2 A\left(S_{1}, S_{2}\right)}{\sqrt{\left|V^{\prime \prime}(0)\right| / I}}\left[\sqrt{\frac{\beta^{2}}{4}+\left|V^{\prime \prime}(0) / I\right|}-\frac{\beta}{2}\right] \\
& \times\left[\tau_{\mathrm{TST}}^{-1}\left(\phi_{1}\right)+\tau_{\mathrm{TST}}^{-1}\left(\phi_{2}\right)\right],
\end{aligned}
$$

where

$$
A\left(S_{1}, S_{2}\right)=\varphi_{1}^{+}(i / 2)-\varphi_{2}^{+}(i / 2)=\varphi^{+}(i / 2) .
$$

Here we have introduced the function $\varphi(\lambda)=\varphi_{1}(\lambda)$ $-\varphi_{2}(\lambda)$. An equation for $\varphi(\lambda)$ can be obtained by subtracting Eq. (A4) from Eq. (A3). We have

$$
\varphi^{+}(\lambda)+\frac{G_{1}(\lambda) G_{2}(\lambda)}{G_{12}(\lambda)} \varphi^{-}(\lambda)=0,
$$

where

$$
\begin{aligned}
G_{12}(\lambda) & =G_{1}(\lambda)+G_{2}(\lambda)-G_{1}(\lambda) G_{2}(\lambda) \\
& =1-\exp \left[-\frac{\beta\left(S_{1}+S_{2}\right)\left(4 \lambda^{2}+1\right)}{4 k T}\right] .
\end{aligned}
$$

Equation (A8) is now in a form whereby one may apply the Wiener-Hopf method. In order to use the method we rewrite Eq. (A8) as

$$
\begin{aligned}
\ln \left[-\varphi^{+}(\lambda)\right]= & \ln G_{1}(\lambda)+\ln G_{2}(\lambda)-\ln G_{12}(\lambda) \\
& +\ln \varphi^{-}(\lambda) .
\end{aligned}
$$

The function $G_{i}(\lambda)$ may now be decomposed as

$$
G_{i}(\lambda)=G_{i}^{+}(\lambda) G_{i}^{-}(\lambda),
$$

where by Cauchy's theorem

$$
G_{i}^{ \pm}(\lambda)=\exp \left[ \pm \frac{1}{2 \pi i} \int_{-\infty}^{\infty} \frac{\ln G_{i}\left(\lambda^{\prime}\right)}{\lambda^{\prime}-\lambda \mp i 0} d \lambda^{\prime}\right] .
$$

Equation (A10) thus has the form

$$
\begin{aligned}
\ln [- & \left.\varphi^{+}(\lambda)\right]-\ln G_{1}^{+}(\lambda)-\ln G_{2}^{+}(\lambda)+\ln G_{12}^{+}(\lambda) \\
& =\ln G_{1}^{-}(\lambda)+\ln G_{2}^{-}(\lambda)-\ln G_{12}^{-}(\lambda)+\ln \varphi^{-}(\lambda) .
\end{aligned}
$$

The functions $f_{1}$ and $f_{2}$ must satisfy the boundary conditions that deep in the wells these distributions become MaxwellBoltzmann distributions so that

$$
f_{i}(E) \sim \frac{\sqrt{V^{\prime \prime}\left(\phi_{i}\right) / I}}{2 \pi k T} e^{-\left[E-V\left(\phi_{i}\right)\right] /(k T)}, \quad-E \gg k T .
$$

As a consequence the functions $\varphi_{i}^{ \pm}(\lambda)$ from Eq. (A1) have poles (the choice of the prefactor in that equation should now be evident)

$$
\varphi_{i}^{ \pm}(\lambda)=\frac{-i}{\lambda \pm i / 2}, \quad|\lambda+i / 2| \ll 1 .
$$

As the functions in the left- and in the right-hand side of Eq. (A13) are analytic in different half planes for complex $\lambda$ they should equal an entire function, which satisfies Eq. (A15), ${ }^{11}$

$$
\varphi^{+}(\lambda)=i \frac{G_{1}^{-}(-i / 2) G_{2}^{-}(-i / 2) G_{1}^{+}(\lambda) G_{2}^{+}(\lambda)}{(\lambda+i / 2) G_{12}^{+}(-i / 2) G_{12}^{-}(\lambda)} .
$$

Hence in Eq. (A6)

$$
A\left(S_{1}, S_{2}\right)=\frac{A^{\prime}\left(S_{1}\right) A^{\prime}\left(S_{2}\right)}{A^{\prime}\left(S_{1}+S_{2}\right)},
$$

where $A^{\prime}\left(S_{i}\right)$ is related to the Fourier transform of the Green function by

$$
A^{\prime}\left(S_{i}\right)=\left|G_{i}^{+}(i / 2)\right|^{2} .
$$

Substituting $G_{i}^{+}$from Eq. (A12) into Eq. (A18) and noting Eqs. (22) and (31), one obtains the function $A^{\prime}(\Delta)$ in Eq. (30) bridging the VLD and TST results.

\section{APPENDIX B: EQUATIONS FOR $\mathrm{C}_{1}(0), \mathrm{Q}_{n}^{+}$, AND $\mathrm{Q}_{n}^{-}$}

The column vector $\mathbf{C}_{1}(0)$ and the matrices $\mathbf{Q}_{n}^{+}$and $\mathbf{Q}_{n}^{-}$ are given by 


$$
\begin{aligned}
& \mathbf{C}_{1}(0)=\frac{i}{2 I_{0}(\sigma)}\left|\begin{array}{c}
\vdots \\
I_{2}(\sigma)+I_{3}(\sigma) \\
0 \\
-I_{2}(\sigma)-I_{1}(\sigma) \\
0 \\
I_{0}(\sigma)+I_{1}(\sigma) \\
-I_{0}(\sigma)-I_{1}(\sigma) \\
0 \\
I_{2}(\sigma)+I_{1}(\sigma) \\
0 \\
-I_{2}(\sigma)-I_{3}(\sigma) \\
\vdots
\end{array}\right| \\
& \mathbf{Q}_{n}^{+}=-\frac{i}{2}\left(\begin{array}{ccccccc}
\ddots & \vdots & \vdots & \vdots & \vdots & \vdots & . \\
\cdots & -2 & 0 & 0 & 0 & 0 & \cdots \\
\cdots & 0 & -1 & 0 & 0 & 0 & \cdots \\
\cdots & 0 & 0 & 0 & 0 & 0 & \cdots \\
\cdots & 0 & 0 & 0 & 1 & 0 & \cdots \\
\cdots & 0 & 0 & 0 & 0 & 2 & \cdots \\
\cdot & \vdots & \vdots & \vdots & \vdots & \vdots & \ddots
\end{array}\right) \\
& \mathbf{Q}_{n}^{-}=-i(n-1)\left(\begin{array}{ccccccccccc}
\ddots & \vdots & \vdots & \vdots & \vdots & \vdots & \vdots & \vdots & \vdots & \vdots & . \\
\cdots & \sigma & 0 & -2 & 0 & -\sigma & 0 & 0 & 0 & 0 & \cdots \\
\cdots & 0 & \sigma & 0 & -1 & 0 & -\sigma & 0 & 0 & 0 & \cdots \\
\cdots & 0 & 0 & \sigma & 0 & 0 & 0 & -\sigma & 0 & 0 & \cdots \\
\cdots & 0 & 0 & 0 & \sigma & 0 & 1 & 0 & -\sigma & 0 & \cdots \\
\cdots & 0 & 0 & 0 & 0 & \sigma & 0 & 2 & 0 & -\sigma & \cdots \\
\cdot & \vdots & \vdots & \vdots & \vdots & \vdots & \vdots & \vdots & \vdots & \vdots & \ddots
\end{array}\right)
\end{aligned}
$$

The exceptions are the matrices $\mathbf{Q}_{1}^{+}$and $\mathbf{Q}_{2}^{-}$, which are given by

$$
\begin{aligned}
& \mathbf{Q}_{1}^{+}=-\frac{i}{2}\left(\begin{array}{ccccccc}
\ddots & \vdots & \vdots & \vdots & \vdots & \vdots & . \\
\cdots & -2 & 0 & 0 & 0 & 0 & \cdots \\
\cdots & 0 & -1 & 0 & 0 & 0 & \cdots \\
\cdots & 0 & 0 & 0 & 1 & 0 & \cdots \\
\cdots & 0 & 0 & 0 & 0 & 2 & \cdots \\
\cdot & \vdots & \vdots & \vdots & \vdots & \vdots & \ddots
\end{array}\right), \\
& \mathbf{Q}_{2}^{-}=-i\left(\begin{array}{cccccccccc}
\ddots & \vdots & \vdots & \vdots & \vdots & \vdots & \vdots & \vdots & \vdots & . \\
\cdots & \sigma & 0 & -2 & 0 & 0 & 0 & 0 & 0 & \cdots \\
\cdots & 0 & \sigma & 0 & -1 & -\sigma & 0 & 0 & 0 & \cdots \\
\cdots & 0 & 0 & \sigma & 0 & 0 & -\sigma & 0 & 0 & \cdots \\
\cdots & 0 & 0 & 0 & \sigma & 1 & 0 & -\sigma & 0 & \cdots \\
\cdots & 0 & 0 & 0 & 0 & 0 & 2 & 0 & -\sigma & \cdots \\
. & \vdots & \vdots & \vdots & \vdots & \vdots & \vdots & \vdots & \vdots & \ddots
\end{array}\right) .
\end{aligned}
$$




\section{APPENDIX C: EVALUATION OF AVERAGES IN THE UNDAMPED LIMIT}

In the very low damping limit $\left(\beta^{\prime} \ll 1\right)$, the energy of the dipole is not conserved but will vary very slowly with time (quasistationarity). Thus the dynamics of the system are described by the one-dimensional Fokker-Planck equation (55) and differ but little from those of the undamped limit $\left(\beta^{\prime}=0\right)$. In the undamped limit [when the Langevin torque in
Eq. (7) vanishes], the energy $\varepsilon$, Eq. (54), is a constant of the motion. Thus the dynamics of the dipole are described by the deterministic nonlinear differential equation

$$
\eta \frac{d}{d t} \phi(t)= \pm \sqrt{\varepsilon+2 \sigma \sin ^{2} \phi(t)} .
$$

Equation $(\mathrm{C} 1)$ has a solution ${ }^{45}$ in terms of the Jacobian doubly periodic elliptic function $\operatorname{cn}(u \mid m)$ and $\operatorname{dn}(u \mid m):^{31}$

$$
\sin \phi(t)=\left\{\begin{array}{l} 
\pm \operatorname{dn}\left(\frac{t}{\eta} \sqrt{2 \sigma}+w \mid m(\varepsilon)\right), \quad-2 \sigma \leqslant \varepsilon<0, \\
\pm \operatorname{cn}\left(\frac{t}{\eta} \sqrt{\varepsilon+2 \sigma}+w \sqrt{m(\varepsilon)} \mid m^{-1}(\varepsilon)\right), \quad 0 \leqslant \varepsilon<\infty,
\end{array},\right.
$$

where

$$
w=\int_{0}^{\phi(0)}\left[1-m(\varepsilon) \sin ^{2} x\right]^{-1 / 2} d x, \quad m(\varepsilon)=1+\varepsilon / 2 \sigma .
$$

By noting that $\operatorname{sn}^{2}(u \mid m)+\mathrm{cn}^{2}(u \mid m)=1$ and $m \operatorname{sn}^{2}(u \mid m)+\operatorname{dn}^{2}(u \mid m)=1,{ }^{31}$ one has

$$
\sin ^{2} \phi(t)=\left\{\begin{array}{l}
1-m(\varepsilon) \operatorname{sn}^{2}\left(\frac{t}{\eta} \sqrt{2 \sigma}+w \mid m(\varepsilon)\right), \quad-2 \sigma \leqslant \varepsilon<0, \\
1-\operatorname{sn}^{2}\left(\frac{t}{\eta} \sqrt{\varepsilon+2 \sigma}+w \sqrt{m(\varepsilon)} \mid m^{-1}(\varepsilon)\right), \quad 0 \leqslant \varepsilon<\infty,
\end{array},\right.
$$

In order to proceed, we recall the Fourier series for the Jacobi functions ${ }^{46}$

$$
\begin{gathered}
\operatorname{cn}(u \mid m)=\frac{2 \pi}{m^{1 / 2} K(m)} \sum_{n=0}^{\infty} \frac{q^{n+1 / 2}}{1+q^{2 n+1}} \cos \left[\frac{(2 n+1) \pi u}{2 K(m)}\right], \\
\operatorname{dn}(u \mid m)=\frac{\pi}{2 K(m)}+\frac{2 \pi}{K(m)} \sum_{n=1}^{\infty} \frac{q^{n}}{1+q^{2 n}} \cos \left[\frac{n \pi u}{K(m)}\right], \\
m \operatorname{sn}^{2}(u \mid m)=1-\frac{E(m)}{K(m)}-\frac{2 \pi^{2}}{K^{2}(m)} \\
\quad \times \sum_{n=1}^{\infty} \frac{n q^{n}}{1-q^{2 n}} \cos \left[\frac{n \pi u}{K(m)}\right],
\end{gathered}
$$

where $q=\exp [-\pi K(1-m) / K(m)]$. Thus, from Eqs. (C3)(C6), we can readily obtain $\overline{\overline{\sin \phi}}$ and $\overline{\overline{\sin ^{2} \phi}}$ averaged over the phase $w$, viz.,

$$
\begin{aligned}
\overline{\overline{\sin \phi(\varepsilon, w}} & =\frac{1}{4 K} \int_{-2 K}^{2 K} \sin \phi(\varepsilon, w) d w \\
& = \begin{cases} \pm \pi /(2 K[m(\varepsilon)]), \quad-2 \sigma \leqslant \varepsilon<0 . \\
0, & 0 \leqslant \varepsilon<\infty\end{cases}
\end{aligned}
$$

$$
\begin{aligned}
\overline{\overline{\sin ^{2} \phi(\varepsilon, w}} & =\frac{1}{4 K} \int_{-2 K}^{2 K} \sin ^{2} \phi(\varepsilon, w) d w \\
& = \begin{cases}\frac{E[m(\varepsilon)]}{K[m(\varepsilon)]}, & -2 \sigma \leqslant \varepsilon<0, \\
1-m(\varepsilon)\left\{1-\frac{E\left[m^{-1}(\varepsilon)\right]}{K\left[m^{-1}(\varepsilon)\right]},\right. & 0 \leqslant \varepsilon<\infty .\end{cases}
\end{aligned}
$$

Accordingly, on noting that $W_{\text {st }}$ is the equilibrium MaxwellBoltzmann distribution $W_{0}$, viz.,

$W_{0}[\phi(0), \dot{\phi}(0)] d \phi(0) d \dot{\phi}(0)$

$$
=\frac{\eta e^{-\sigma}}{2 \pi^{3 / 2} I_{0}(\sigma)} e^{-\eta^{2} \dot{\phi}^{2}(0)+2 \sigma \sin ^{2} \phi(0)} d \phi(0) d \dot{\phi}(0),
$$

by making the transformation of the variables $\{\phi(0), \dot{\phi}(0)\}$ $\rightarrow\{w, \varepsilon\},{ }^{45}$ and by integrating the distribution function $W_{0}(\varepsilon)$ over the phase $w$, we have

$W_{0}(\varepsilon) d \varepsilon$

$$
\begin{gathered}
=\frac{\sqrt{2} e^{-\sigma}}{\pi^{3 / 2} \sigma^{1 / 2} I_{0}(\sigma)} \operatorname{Re}\{K[m(\varepsilon)]\} e^{-\varepsilon} d \varepsilon, \\
\int_{-2 \sigma}^{\infty} W_{0}(\varepsilon) d \varepsilon=1 .
\end{gathered}
$$

The average of a dynamical quantity $\overline{\overline{A(\varepsilon, w}}$ ) is defined as 


$$
\begin{aligned}
\langle\overline{\bar{A}}\rangle_{0}= & \int_{-2 \sigma}^{\infty} \overline{\bar{A}}(\varepsilon) W_{0}(\varepsilon) d \varepsilon \\
= & \frac{\sqrt{2} e^{-\sigma}}{\pi^{3 / 2} \sigma^{1 / 2} I_{0}(\sigma)}\left[\int_{-2 \sigma}^{0} \overline{\bar{A}}(\varepsilon) K[m(\varepsilon)] e^{-\varepsilon} d \varepsilon\right. \\
& \left.+\int_{0}^{\infty} \frac{1}{\sqrt{1+\varepsilon / 2 \sigma}} \overline{\bar{A}}(\varepsilon) K\left[m^{-1}(\varepsilon)\right] e^{-\varepsilon} d \varepsilon\right] .
\end{aligned}
$$

(C10)

In particular, we have from Eqs. (C8) and (C10)

$$
\begin{aligned}
\left\langle\overline{\overline{\sin ^{2}}} \phi\right\rangle_{0} & =\int_{-2 \sigma}^{\infty} \overline{\overline{\sin ^{2}}} \phi(\varepsilon) W_{0}(\varepsilon) d \varepsilon \\
& =\frac{\sqrt{2} e^{-\sigma}}{\pi^{3 / 2} \sigma^{1 / 2} I_{0}(\sigma)} \int_{-2 \sigma}^{\infty} \operatorname{Re}\{E[m(\varepsilon)]\} e^{-\varepsilon} d \varepsilon
\end{aligned}
$$

(C11)

Equation (C11) yields the same equilibrium value as Eq. (53). One can also verify that the equipartition theorem, viz.,

$$
\eta^{2}\left\langle\overline{\overline{\dot{\phi}^{2}}}\right\rangle_{0}=\left\langle\varepsilon+2 \sigma \overline{\overline{\sin ^{2}}} \phi\right\rangle_{0}=\frac{1}{2},
$$

also holds. By using Eqs. (C7)-(C11), we have Eq. (56).

The longitudinal correlation function $C(t)$ $=\langle\sin \phi(0) \sin \phi(t)\rangle_{0}$ for the free rotation can be derived from Eqs. (C2), (C4), (C5), and (C9) and is given by

$$
\begin{aligned}
C(t)= & \frac{2 \sqrt{2 \pi / \sigma}}{I_{0}(\sigma)+I_{1}(\sigma)} \\
& \times\left\{\int_{-2 \sigma}^{0}\left(\frac{1}{8}+\sum_{n=1}^{\infty} \frac{q^{2 n}}{\left(1+q^{2 n}\right)^{2}} \cos \left[\frac{n \pi \sqrt{2 \sigma}}{\eta K[m(\varepsilon)]} t\right]\right)\right. \\
& \times \frac{e^{-\varepsilon-\sigma}}{K[m(\varepsilon)]} d \varepsilon+\int_{0}^{\infty} \frac{m^{1 / 2}(\varepsilon) e^{-\varepsilon-\sigma}}{K\left[m^{-1}(\varepsilon)\right]} \\
& \left.\times \sum_{n=1}^{\infty} \frac{q^{2 n-1}}{\left(1+q^{2 n-1}\right)^{2}} \cos \left[\frac{(2 n-1) \pi \sqrt{\varepsilon+2 \sigma}}{2 \eta K\left[m^{-1}(\varepsilon)\right]} t\right] d \varepsilon\right\} .
\end{aligned}
$$

For $\sigma=0, C(t)$ from Eq. (C13) reduces to the free rotator correlation function, viz.,

$$
C(t)=\frac{1}{\sqrt{\pi}} \int_{0}^{\infty} \frac{1}{\sqrt{\varepsilon}} \cos (\sqrt{\varepsilon} t / \eta) e^{-\varepsilon} d \varepsilon=e^{-t^{2} / 4 \eta^{2}} .
$$

Equation (C14) yields $\tau_{\|}=\eta \sqrt{\pi}$.
${ }^{1}$ H. Risken, The Fokker-Planck Equation, 2nd Ed. (Springer, Berlin, 1989).

${ }^{2}$ W. T. Coffey, Yu. P. Kalmykov, and J. T. Waldron, The Langevin Equation, 2nd Ed. (World Scientific, Singapore, 2004).

${ }^{3}$ G. Barone and A. Paterno, Physics and Applications of the Josephson Effect (Wiley, New York, 1982).

${ }^{4}$ Yu. P. Kalmykov, Phys. Rev. E 61, 6320 (2000); 62, 227 (2000).

${ }^{5}$ H. A. Kramers, Physica (Utrecht) 7, 284 (1940).

${ }^{6}$ P. Hänggi, P. Talkner, and M. Borkovec, Rev. Mod. Phys. 62, 251 (1990).

${ }^{7}$ W. T. Coffey, D. A. Garanin, and D. McCarthy, Adv. Chem. Phys. 117, 528 (2001).

${ }^{8}$ V. I. Mel'nikov, Physica A 130, 606 (1985); Phys. Rep. 209, 1 (1991).

${ }^{9}$ V. I. Mel'nikov and S. V. Meshkov, J. Chem. Phys. 85, 1018 (1986).

${ }^{10}$ H. Grabert, Phys. Rev. Lett. 61, 1683 (1988).

${ }^{11}$ E. Pollak, H. Grabert, and P. Hänggi, J. Chem. Phys. 91, 4073 (1989).

${ }^{12}$ I. Rips and E. Pollak, Phys. Rev. A 41, 5366 (1990).

${ }^{13}$ M. Topaler and N. Makri, J. Chem. Phys. 101, 7500 (1994).

${ }^{14}$ A. N. Drozdov and P. Talkner, J. Chem. Phys. 109, 2080 (1998).

${ }^{15}$ R. Ferrando, R. Spadacini, and G. E. Tommei, Phys. Rev. A 46, R699 (1992).

${ }^{16}$ R. Ferrando, R. Spadacini, and G. E. Tommei, Phys. Rev. E 48, 2437 (1993).

${ }^{17}$ E. Pollak, J. Bader, B. J. Berne, and P. Talkner, Phys. Rev. Lett. 70, 3299 (1993).

${ }^{18}$ Yu. Georgievskii and E. Pollak, Phys. Rev. Lett. 70, 5098 (1994).

${ }^{19}$ E. Hershkovitz, P. Talkner, E. Pollak, and Yu. Georgievskii, Surf. Sci. 421, 73 (1999).

${ }^{20}$ R. W. Pastor and A. Szabo, J. Chem. Phys. 97, 5098 (1992).

${ }^{21}$ P. M. Déjardin, D. S. F. Crothers, W. T. Coffey, and D. J. McCarthy, Phys. Rev. E 63, 021102 (2001).

${ }^{22}$ J. I. Lauritzen and R. Zwanzig, Jr., Adv. Mol. Relax. Interact. Processes 5, 339 (1973).

${ }^{23}$ W. T. Coffey, Yu. P. Kalmykov, E. S. Massawe, and J. T. Waldron, J. Chem. Phys. 99, 4011 (1993).

${ }^{24}$ C. J. Reid, Mol. Phys. 49, 331 (1983).

${ }^{25}$ F. Marchesoni and J. K. Vij, Z. Phys. B: Condens. Matter 58, 187 (1985); F. Marchesoni, Phys. Rev. B 32, 1827 (1985).

${ }^{26}$ E. Praestgaard and N. G. van Kampen, Mol. Phys. 43, 33 (1981).

${ }^{27}$ W. T. Coffey, M. W. Evans, and P. Grigolini, Molecular Diffusion and Spectra (Wiley, New York, 1984; Russian translation: Mir, Moscow, 1987).

${ }^{28}$ W. T. Coffey, Yu. P. Kalmykov, and S. V. Titov, J. Chem. Phys. 115, 9895 (2001).

${ }^{29}$ W. T. Coffey, Yu. P. Kalmykov, and S. V. Titov, Phys. Rev. E 67, 061115 (2003).

${ }^{30}$ H. Goldstein, Classical Mechanics, 2nd Ed. (Addison-Wesley, Reading, MA, 1980).

${ }^{31}$ Handbook of Mathematical Functions, edited by M. Abramowitz and I. Stegun (Dover, New York, 1964).

${ }^{32}$ R. A. Sack, Proc. R. Soc. London, Ser. B 70, 402 (1957).

${ }^{33}$ V. I. Mel'nikov, Phys. Rev. E 48, 3271 (1993); 50, 627 (1994).

${ }^{34}$ P. Talkner and E. Pollak, Phys. Rev. E 47, R21 (1993).

${ }^{35}$ R. Ferrando, R. Spadacini, G. E. Tommei, and V. I. Mel'nikov, Phys. Rev. E 51, R1645 (1995).

${ }^{36}$ A. Szabo, J. Chem. Phys. 72, 4620 (1980).

${ }^{37}$ C. P. Bean and J. D. Livingston, J. Appl. Phys. 30, 120S (1959).

${ }^{38}$ A. O. Caldeira and A. J. Leggett, Physica A 121, 587 (1983).

${ }^{39}$ C. Anastopoulos and J. J. Halliwell, Phys. Rev. D 51, 6870 (1995).

${ }^{40}$ J. L. Garcia-Palacios, Europhys. Lett. 65, 735 (2004).

${ }^{41}$ U. Weiss, Quantum Dissipative Systems, 2nd Ed. (World Scientific, Singapore, 1999).

42 J. S. Langer, Ann. Phys. (N.Y.) 54, 258 (1969).

${ }^{43}$ R. Becker and W. Döring, Ann. Phys. (Leipzig) 24, 719 (1935).

${ }^{44}$ E. C. Titchmarsh, Introduction to the Theory of Fourier Integrals (Oxford University Press, London, 1937).

${ }^{45}$ Yu. P. Kalmykov, Khim. Fiz. 6, 745 (1987) [Sov. J. Chem. Phys. 6, 1427 (1990)].

${ }^{46}$ E. T. Whittaker and G. N. Watson, A Course of Modern Analysis, 4th Ed. (Cambridge University Press, Cambridge, England, 1927). 